\title{
DIPTERES TORRENTICOLES PEU CONNUS : VII. LES CECIDOMYIIDAE PORRICONDYLINAE DU SUD-OUEST DE LA FRANCE (NEMATOCERA)
}

\author{
par A. G. B. THOMAS ${ }^{1}$
}

19 imagos de Porricondyla ramadei n. sp. ont été obtenues par élevage. Biotope: des touffes de Bryophytes immergées en eau agitée, dans un torrent des Hautes-Pyrénées à $1260 \mathrm{~m}$ d'altitude. Le prélèvement totalisait par ailleurs plusieurs centaines d'invertébrés d'espèces considérées comme franchement torrenticoles.

\section{Poorly known torrential Diptera : VII. Cecidomyiidae Porricondylinae (Nematocera) from South-West of France.}

19 adults of Porricondyla ramadei $n$. sp. have been obtained by rearing from younger stages. The biotope is tufts of submerged Bryophytes in turbulent water in a mountain stream at an altitude of $1260 \mathrm{~m}$ (High-Pyrenees). The sample also contained several hundred invertebrates belonging to strictly torrential species.

\section{I. - INTRODUCTION}

Les Cecidomyiidae sont des Diptères Nématocères de petite taille, surtout connus par leurs larves souvent galligènes ou gallicoles sur de nombreux végétaux (d'où leur nom anglais de "gall-gnats") : certaines espèces, en particulier celles inféodées à des Graminées ou à des Légumineuses, peuvent ainsi présenter une importance économique considérable en agronomie.

En réalité, l'habitat des larves et leur mode de vie sont extrêmement diversifiés : certaines sont prédatrices, d'autres sont parasites de pucerons (Séguy 1955) ou commensales de divers insectes galligènes (Séguy $1951 \mathrm{a}$ ), beaucoup colonisent mousses, hépatiques, champignons, bois pourri, résine de conifères (Séguy 1951 b) ou cônes euxmêmes (Foote 1956) et aussi végétaux aquatiques ou subaquatiques. Ainsi, par exemple, Séguy (1950) rapporte que les larves du genre

1. Laboratoire d'Hydrobiologie (ERA 702), Université Paul-Sabatier, 118, route de Narbonne, 31062 Toulouse Cedex. 
Hormomyia vivent dans les tissus de Carex et, en cas de submersion, respirent l'oxygène libéré à l'intérieur de la galle.

En particulier, quatre genres sont cités dans la Susswässer Fauna Deustschlands par Grünberg (1910) surtout d'après le travail de Rübsaamen (1899). Quatre autres genres sont signalés par Felt (1913, rapporté par Johannsen 1934) de biotopes similaires (plantes aquatiques) en Amérique du Nord. Toutefois, ces citations sont depuis longtemps tombées dans l'oubli : les manuels d'hydrobiologie plus récents ne font aucune mention de la famille des Cecidomyiidae, tant en Europe: Wesenberg-Lund (1943), Illies et coll. (1967, 1978) qu'aux Etats-Unis : Usinger et coll. (1963).

Récemment, Tsacas et Legrand (1979) ont découvert des larves de Bremia sp. dans les pontes, aériennes, d'une espèce d'Odonate africaine. Or, "ces pontes se présentent sous forme d'une masse gélatineuse à l'intérieur de laquelle se trouvent les œufs".

Chez les Cecidomyiidae, une subdivision taxonomique importante, et admise depuis très longtemps, est celle des Porricondyliens. Précisément, les Porricondyliens, érigés en une sous-famille distincte - les Porricondylinae $^{1}$ - surtout après les travaux de Möhn (1955) et de Panelius (1965) présentent, au moins pour la quasi totalité ce leurs espèces, des larves libres "probablement toutes mycophages" (Panelius t.c.) et considérées jusqu'ici comme " terrestres". Séguy (1937) indique que ces larves se rencontrent surtout sous les écorces de bois mort et dans les vieux troncs d'arbres en décomposition (en particulier celles de l'important genre Porricondyla sur le hêtre, le charme et le bouleau). Selon le même auteur (1950), une espèce de ce genre vivrait dans la partie émergée de plants de Phragmites communis. Des imagos de deux espèces de Porricondyla ont été obtenues dans des pièges d'émergence placés sur le sol (Szadziewski 1976).

A ma connaissance, Vaillant (1955) est le seul à avoir mentionné la présence de larves de Cecidomyiidae libres (Hammamyia muscicola et Porricondyla sp.) dans un biotope franchement aquatique (milieu madicole). Mais cet auteur considère que la seconde espèce appartient à la faune commensale terrestre.

La mise en élevage de la faune immature inféodée à des Bryophytes aquatiques m'a permis d'obtenir près de 20 imagos conspécifiques de Porricondylinae. Porricondyla ramadei $\mathrm{n}$. $\mathrm{sp}$. est la première espèce de Cecidomyiidae découverte dans un biotope torrenticole. Elle est dédiée à $M$. F. Ramade, ingénieur agronome, actuellement professeur de Zoologie et d'Ecologie à la Faculté d'Orsay.

1. Ce rang de sous-famille n'a pas été repris par les auteurs d'Europe centrale et orientale comme il l'a été en Europe occidentale, au Japon et aux Etats-Unis (par exemple par: Harris (1966), Yukawa et Nijveldt (1975), Parnell (1971)). 


\section{2. - DESCRIPTION (Planche I)}

\subsection{Mâle}

Coloration d'ensemble : brun clair à roux.

Tête.

Antennes à $2+14$ segments. Scape (fig. 1 a) : 3 ou 4 soies medioventrales; pédicelle subsphérique. Flage!lomères à tige nue et corps couvert de microtriches. Pour le $3^{e}$ flagellomère (fig. $1 \mathrm{~b}$ ) : rapport des longueurs tige/corps $=1,5 ;$ rapport de la longueur du corps à son diamètre (protubérances latérales non comprises) $=1,75$. Un circumfilum en anneau vers le tiers basal de chaque corps de flagellomère, bien visible jusqu'au $8^{c}$ ou $9^{\mathrm{e}}$, ensuite plus ou moins nettement jusqu'au $11^{\text {2 }}$ ou même $12^{\text {e }}$.

Pont interoculaire : 4 à 6 ommatidies de largeur dans la partie la plus étroite.

Palpes à $1+4$ articles de longueur croissante.

Thorax.

3 ou 4 soies mésopleurales fines; 10 à 12 soies dorso-latérales; des soies dorso-centrales et scutellaires.

Longues griffes tarsales bifides (fig. 2), la dent interne très développée ; empodium plumeux de longueur assez réduite.

Ailes (fig. 3) : longueur de 3,1 à $3,8 \mathrm{~mm}$. Rapport de la longueur à la largeur maximale : 2,7. $\mathrm{M}$ à peu près invisible. $\mathrm{Cu}_{2}$ bien marquée, $\mathrm{Cu}_{1}$ estompée, à peu près rectiligne sauf à sa séparation de $\mathrm{Cu}_{2}$. Rapport des distances $\mathrm{Cu}_{1}-\mathrm{Cu}_{2} / \mathrm{Cu}_{1}-\mathrm{R} 5$ en général voisin de 0,85 mais pouvant être nettement inférieur.

Genitalia (fig. $4 \mathrm{a}$ à c).

Hypopyge dirigé vers le haut. Angle ventral supérieur interne des basistyles nettement proéminent (fig. 4 a). Distiforceps de rapport longueur/largeur $=1,35$, arrondi vers l'extérieur; du côté interne, la face supérieure est toutefois nettement aplatie (fig. $4 \mathrm{a}, \mathrm{b}$ et c); le bord interne du distiforceps est frangé de soies denses et courtes. Crochets du tegmen étroits, convergents, recourbés dorsalement de part et d'autre du pénis mais ne se croisant pas. Pénis plus long que les basistyles, très fin sur la moitié distale et au contraire, brusquement très élargi sur la moitié proximale.

\subsection{Femelle}

Antennes: $2+11$ segments. Scape portant 3, parfois 4 soies médioventrales plus longues et plus fortes que chez le mâle et dépassant 


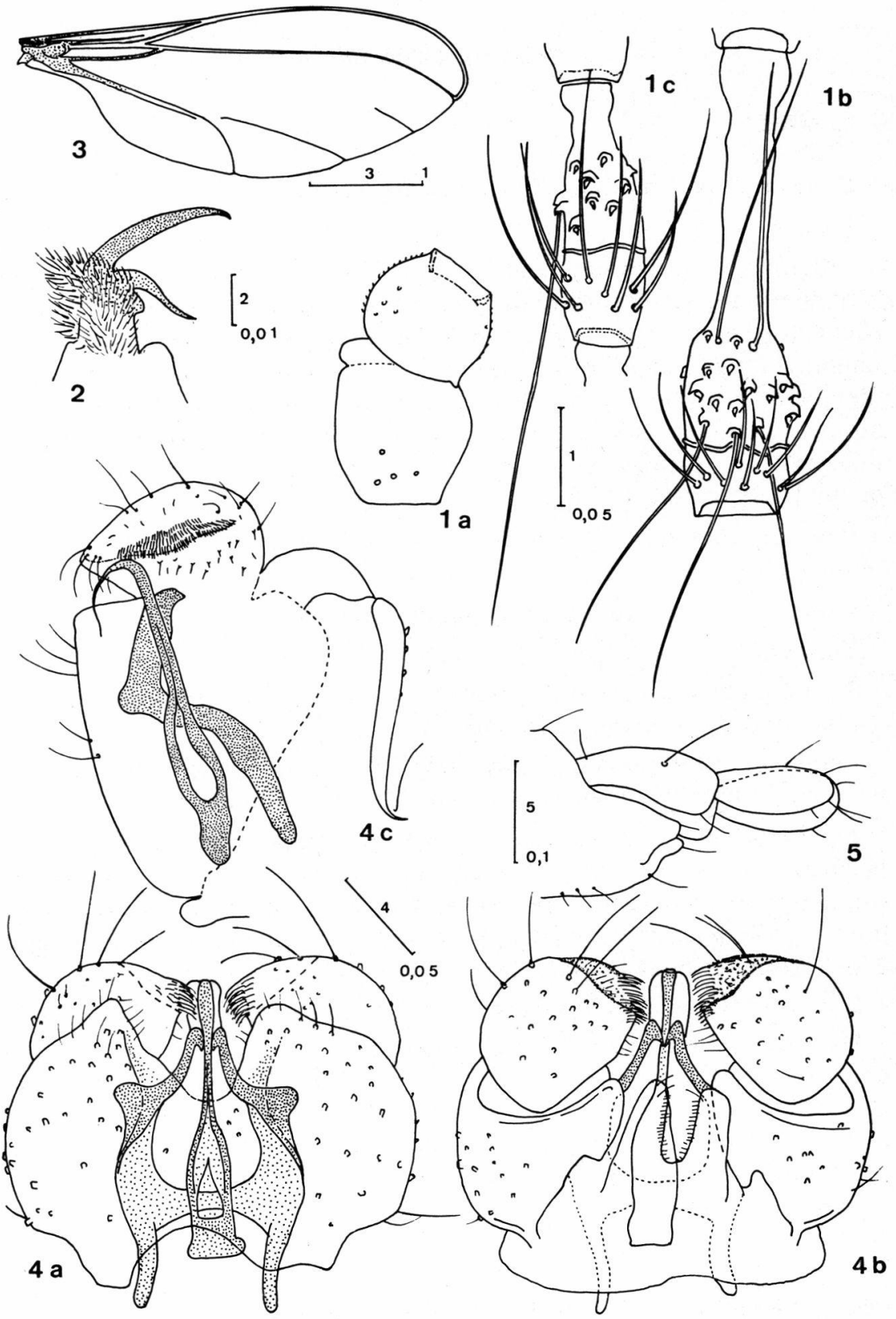

Planche I. - Fig. 1 à 5 : Porricondyla ramadei $\mathrm{n}$. $\mathrm{sp}$. (Echelle en $\mathrm{mm}$.)

f IG. 1: antennes en vue ventrale, antérieure $(1 \mathrm{a}$ : scape et pédicelle du mâle;

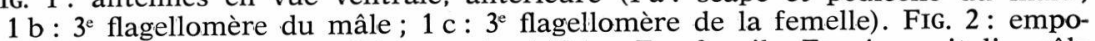
dium et l'une des deux griffes tarsales bifides. FIG. 3 : aile. FIG. 4 : genitalia mâle (vue ventrale, postérieure: $4 \mathrm{a}$; vue dorsale, antérieure: $4 \mathrm{~b}$; vue de profil, demi-forceps droit retiré : 4 c). Fig. 5 : terminalia femelle. 
la base du premier flagellomère. Flagellomères (fig. $1 \mathrm{c}$ ) couverts de microtriches sur les trois-quarts proximaux de leur longueur ou davantage et porteurs, chacun, d'un verticille de soies sub-basales dressées et d'une soie de même orientation, insérée plus haut, sur la face dorsale, postérieure. Les dix premiers flagellomères portent aussi un circumfilum en anneau, tout comme celui du mâle et situé environ à la limite du tiers basal. Il n'existe pas de second anneau en position distale, tout au plus un début de connectif longitudinal qui n'est qu'exceptionnellement visible.

Longueur de l'aile : 3,1 à $4,5 \mathrm{~mm}$; rapport de la longueur à la largeur maximale : 2,9 .

Terminalia (fig. 5).

Les deux lamelles de l'ovipositeur possèdent chacune deux segments bien distincts. Segment distal relativement long - rapport longueur/ largeur maximale : 2 à 2,3 - et arrondi à l'extrémité.

Porricondyla ramadei est, par la morphologie du mâle, une espèce assez proche de $P$. rufocinerea Panelius, 1965 et de $P$. elongata (Felt, 1908) dont femelles et biologie sont inconnues dans les deux cas.

\section{Matériel examiné}

Département des Hautes-Pyrénées :

- la Neste de Couplan à $1260 \mathrm{~m}: 8$ ô (dont l'holotype) et 11 o (dont l'allotype) obtenus par élevage (prélèvement du 17-VII-1975) ;

- le ruisseau de la Mousquère à $1200 \mathrm{~m}: 2$ of et 1 o capturés le 10VIII-1972.

\section{3. - BIOTOPE DES LARVES, AUTRES ESPECES DE LA MEME BIOCENOSE}

Les 19 imagos de $P$. ramadei obtenues par élevage proviennent de Bryophytes aquatiques bordants situés en eau agitée. Plusieurs touffes ont été prélevées près de la surface mais dans la partie immergée, le torrent n'étant alors pas en crue.

N'ont été récoltés dans ce biotope que des spécimens franchement aquatiques:

- 5 individus de Polycelis felina (Planaires);

- 6 larves d'Elodes sp. (Coléoptères, Elodidae);

- 2 exuvies larvaires de Perla grandis (Plécoptères Subulipalpia);

- 1 exuvie larvaire de Leuctra sp. (Plécoptères Filipalpia).

L'élevage des stades immatures pendant deux semaines a, par ailleurs, permis l'émergence des imagos suivantes : 
- 1 \% de Protonemura intricata, 1 d'Amphinemura sulcicollis, $1 \%$ de Leuctra alosi (Plécoptères Filipalpia) ;

- $3 q$ de Sericostoma personatum (Trichoptères) dont la nymphose a lieu sous l'eau;

- 1 \& de Simuliidae ;

- une quinzaine de Chironomidae appartenant aux espèces suivantes: Limnophyes prolongatus, Eukiefferiella coerulescens et Brillia (?) longifurca (\$) ;

- 316 ô et $q$ de Psychodidae du genre Berdeniella; les $\hat{\delta}$ d'un sous échantillonnage de 20 individus appartiennent aux espèces suivantes : B. unispinosa (11), B. cf. cambuerina (7) et B. longispinosa (2) ;

- 1 o de Liancalus virens (Dolichopodidae) ;

- 1 Limoniidae, 1 Phoridae, 6 Cypselidae et 1 Muscidae s.l.

Les espèces identifiées font toutes exclusivement partie du rhithron ou du crénon (Illies et coll. 1978) - en particulier Berdeniella unispinosa, caractéristique des mousses balayées par les remous dans les torrents (F. Vaillant comm. verb.) - à l'exception de Limnophyes prolongatus dont les larves peuvent se rencontrer aussi dans la terre humide (J. N. Tourenq comm. verb.). Une quantité non négligeable de sédiment était en effet retenue par la base des brins de Bryophytes prélevés.

Je remercie les spécialistes qui se sont chargés des déterminations spécifiques: MM. C. Berthélemy (Plécoptères), H. Décamps (Trichoptères), J. N. Tourenq (Chironomidae) et F. Vaillant (Psychodidae).

\section{TRAVAUX CITES}

FELT (E. P.). 1913. - Gall midges in an aquatic or semiaquatic environment. $N$. $Y$. Ent. Soc. J., $21: 62-63$ (non vu).

Foore (R. H.). 1956. - Gall midges associated with cones of western forest trees (Diptera, Itonididae). J. Wash. Acad. Sci., $46: 48-57$ (non vu).

GrüNBERG (K.). 1910. - Diptera, Zweiflügler. In Brauer: Die Sïsswasserfauna Deutschlands. 2A, 312 p. Jena.

JARRIS (K. M.). 1966. - Gall midge genera of economic importance (Diptera : Cecidomyiidae). Part 1: Introduction and subfamily Cecidomyiinae; supertribe Cecidomyiidi. Trans. R. ent. Soc. Lond., 118 (10) : 313-358.

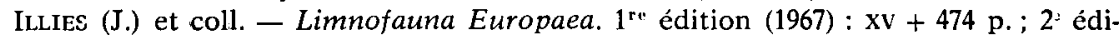
tion (1978): XVII + 532 p. Stuttgart.

JohnNnsen (O. A.). 1934. - Aquatic Diptera. Part I. Nemocera, exclusive of Chironomidae and Ceratopogonidae. Mem. Cornell Univ. agric. Exp. Stn, 164 : $2-71+24 \mathrm{pl}$.

MöHN (E.). 1955. - Beiträge zür Systematik der Larven der Itonididae (= Cecidomyiidae, Diptera) 1 Teil : Porricondylinae und Itonidinae Mitteleuropas. Zoologica, Stuttgart, 38 (105) : $1.247+30 \mathrm{pl}$.

Fanelius (S.). 1965. - A revision of the european gall midges of the subfamily Porricondylinae (Diptera : Itonididae). Acta zool. fenn., 113 : 1-157. 
FARNELl (J. R.). 1971. - A revision of the nearctic Porricondylinae (Diptera: Cecidomyiidae) based largely on an examination of the Felt types. Misc. Publs ent. Soc. Am., $7: 275-348$.

Rübsaamen (E. H.). 1899. - Gallmücken auf Carex und Iris. Wien. ent. Ztg, 18 : $57-76+1 \mathrm{pl}$.

SÉGUY (E.). 1937. - Diptères. In : La Faune de la France, 8: 216 p. Delagrave. Paris.

ŚÉGUY (E.). 1950. - La Biologie des Diptères. 609 p. + 10 pl. Lechevalier. Paris.

St́guy (E.). 1951 a. - Ordre des Diptères, in P. P. Grassé : Traité de Zoologie, 10 (1) : 449-744. Paris.

SÉGuY (E.). 1951 b. - Atlas des Diptères de France, Belgique, Suisse. I. Introduction et caractères généraux, Nématocères, Brachycères $I$. N. Boubée et $\mathrm{C}^{\mathrm{ie}}$. 175 p. +12 pl. Paris.

SÉGuY (E.). 1955. - Introduction à l'étude biologique et morphologique des Insectes Diptères. Publções Mus. nac. Rio de J. : 1-260.

Szadziewski (R.). 1976. - Notes on gall midges (Cecidomyiidae, Diptera) from Poland. I. Polskie Pismo ent., 46: 729-743.

TSACAS (L.) \& LEGRAND (J.). 1979. - Les pontes d'Odonates, gîte larvaire nouveau pour une Drosophile africaine inédite: Drosophila libellulosa, n. sp. (Odonata: Libellulidae; Diptera: Drosophilidae). Rev. fr. Ent., (N.S.), 1 (1) : 13-2\%.

Usinger (R. L.) et coll. 1963. - Aquatic Insects of California. 508 p. Berkeley and Los Angeles.

Villlant (F.). 1955. - Recherches sur la faune madicole de France, de Corse et d'Afrique du Nord. Mém. Mus. natn. Hist. nat., Paris, série A, 11: 1-252 $+6 \mathrm{pl}$.

Wesenderg-Lund (C.). 1943. - Biologie der Süsswasserinsekten. 682 p. Nordisk Forlag. Kopenhagen.

Yukawa (J.) et NiJveldT (W.). 1975. - Redescriptions of two Porricondyline gall midges originally described by De Meijere (Diptera, Cecidomyiidae). Bull. zool. Mus. Univ. Amsterdam, 4 (13) : 103-109. 